\title{
Correction des erreurs dans I'ADN : de la génétique bactérienne aux mécanismes de prédisposition héréditaire aux cancers chez I'homme
}

\author{
Miroslav Radman, François Taddei, Jennifer Halliday
}

"Peut-être que la leçon la plus pertinente de ces quarante dernières années est que les différentes formes que prend la vie sont construites sur des mécanismes essentiellement similaires. On découvre que toutes les espèces ont plus en commun que leurs différences ne le suggèrent ”

John Maddox

à l'occasion du $40^{\mathrm{e}}$ anniversaire de la découverte de la structure de l'ADN. Nature $1993 ; 363$ : 13.

\begin{abstract}
Une chronologie scientifique de l'identification la plus rapide d'un gène
\end{abstract}

D'après le journal Science du 18 mars 1994 , il n'a fallu que "quelques minutes" pour identifier le deuxième gène humain hMLHI (human mutL homologue 1) impliqué dans la prédisposition héréditaire aux cancers du côlon (et plusieurs autres organes), appelée HNPCC (hereditary non polyposis colorectal cancer ou syndrome de Lynch II), alors que pour le premier gène responsable d'HNPCC, hMSH2 (human mutS homologue 2), il aura fallu quelques mois [1]. En ce qui concerne le gène responsable d'autres maladies héréditaires telles que la mucoviscidose ou la maladie de Huntington, il a fallu une dizaine d'années. D'où vient cette accélération des découvertes ? À lire le commentaire cité dans Science, elle serait exclusivement due au développement technologique. En fait, l'extrême rapidité de cette identification tient à deux raisons : (1) la disponibilité récente d'une banque de séquences humaines exprimées (ADNc) et (2) les résultats recherche fondamentale, très sophistiquée, sur les mécanismes de la réparation des mésappariements des bases dans l'ADN chez les bactéries ([2] et figure 1). Grâce à la génétique moléculaire bactérienne, la " chasse " aux gènes d'HNPCC était une recherche " ciblée ", c'est-à-dire que l'on savait ce que l'on cherchait : les gènes humains homologues aux gènes mutS et mutL bactériens. Et on les a identifiés rapidement : en quelques mois par le clonage par PCR ( $h M S H 2)$ et en quelques minutes par le clonage "par téléphone" $(h M L H 1)$. Nous voulons offrir ici un commentaire scientifique sur ces découvertes récentes en présentant ce qui devrait rester après la dissipation de l'émoi médiatique.

Réparation des mésappariements et prédisposition héréditaire au cancer

Après avoir en vain testé des gènes candidats comme RAS ou p53, les chercheurs ont essayé de localiser des gènes responsables du HNPCC avec des marqueurs microsatellites (fournis par l'équipe du Généthon dirigée par J. Weissenbach). Ils ont observé dans les tumeurs HNPCC une forte variabilité de la taille (effet "accordéon") de ces marqueurs génétiques composés de motifs de une à quatre paires de bases répétées de nombreuses fois [3]. Ces instabilités génétiques des répétitions simples observées dans les tumeurs de malades HNPCC rappelaient fortement les observations faites au préalable sur les mutants du système de réparation des mésappariements (SRM) de la bactérie et de la levure.

Au cours de la réplication de l'ADN, les polymérases font fréquemment des erreurs d'alignement sur les séquences répétées en tandem simples ("glissements"). Les structures ainsi formées (bases non appariées ou mal appariées) sont reconnues et réparées par les enzymes du SRM (les protéines MutS, MutL, MutU et MutH, voir [2, 4, 5] pour revue et la figure 2). L'instabilité des microsatellites était donc vraisemblablement due à un défaut dans la fidélité de réplication, plutôt qu'à un défaut dans un gène de contrôle du cycle cellulaire (de type p53). L'observation, chez une levure mutée dans le gène $\mathrm{MSH} 2$ ( $\mathrm{mutS}$ homologue, identifié par PCR grâce à cette 


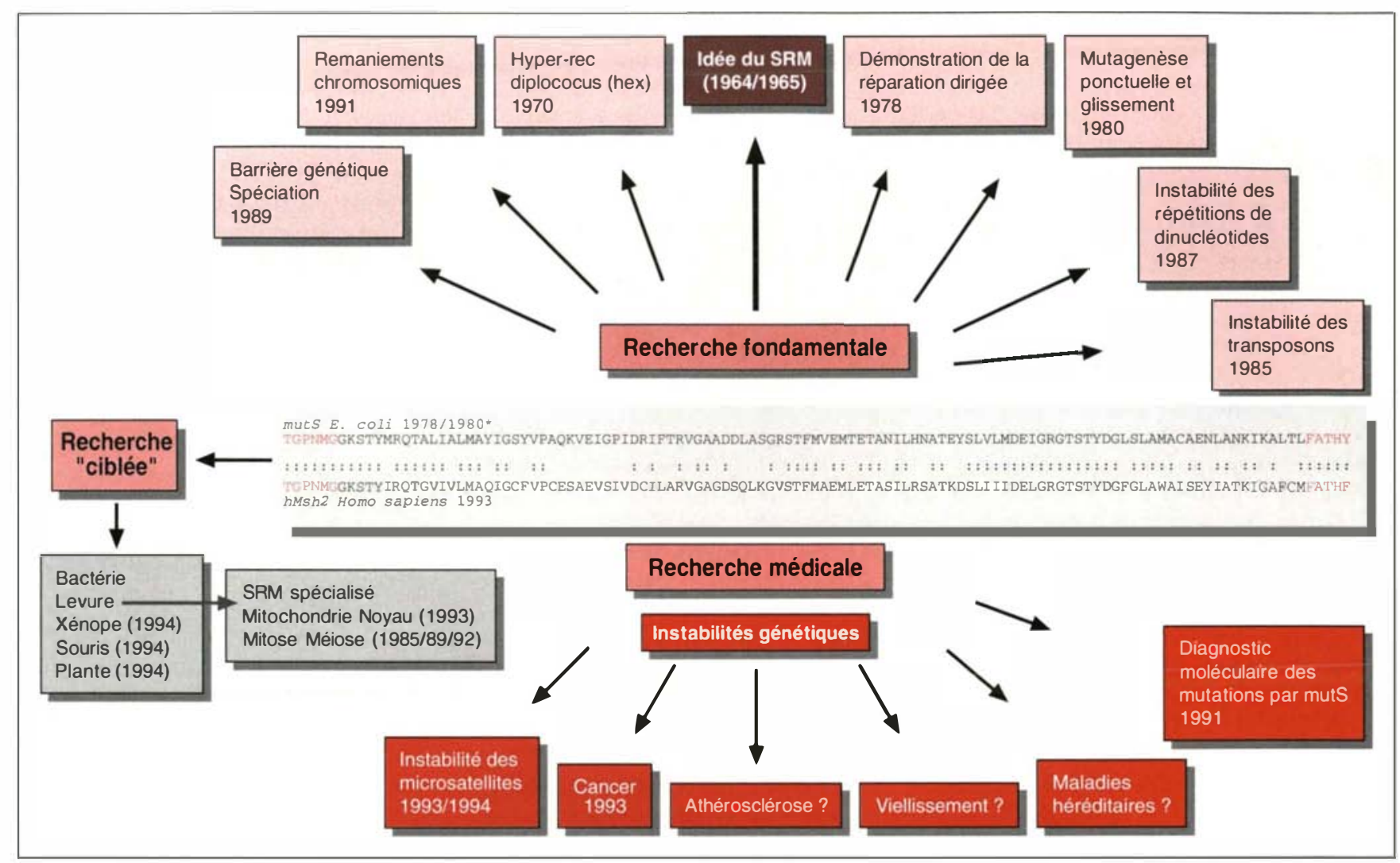

Figure 1. Du système de réparation des mésappariements (SRM) chez la bactérie aux maladies génétiques chez I'homme : une chronologie. Le centre de la figure présente une partie de l'alignement des séquences de mutS d' $\mathrm{E}$. coli et de son homologue humain hMSH2. Les ": "indiquent les acides aminés similaires. Les amorces utilisées dans les réactions de PCR ont été définies à partir des acides aminés représentés en rouge $([6,8-10]$ et Cerovic et Radman, résultats non publiés). Les années entre parenthèses indiquent les dates des observations les plus importantes dans le développement de ce domaine de la génétique moléculaire, les références précises seront trouvées dans le texte. L'association entre le SRM et le vieillissement ou l'athérosclérose est hypothétique mais suggérée par les associations entre la mutagenèse et ses effets cliniques [52].

homologie [6]), d'une instabilité de microsatellites (CA) similaire à celle observée dans les tumeurs HNPCC, renforça cette prédiction [7]. La figure 1 montre la conservation des séquences des gènes codant pour la protéine qui reconnaît les mésappariements des bases dans l'ADN, l'un (mutS) provenant de la bactérie Escherichia coli et l'autre (hMSH2) provenant du génome humain. Une réaction de PCR avec les amorces préalablement utilisées pour identifier les gènes de levure [6], de xénope et de souris (I. Varlet, M. Radman, N.D. Wind, 1994, résultats non publiés) permit alors d'isoler le gène hMSH2, localisé au locus attendu sur le chromosome 2 . Ainsi, en décembre 1993, deux équipes dirigées par B. Vogelstein (Johns Hopkins School of Medicine à Baltimore, USA) et par R. Kolodner (Dana-Farber Cancer Institute à Boston, USA) publièrent la séquence d'un gène nommé hMSH2 responsable de $60 \%$ des cas héréditaires de cancer du côlon (HNPCC) [8-10].

Au mois de mars 1994, ces deux mêmes équipes ( $\mathrm{R}$. Kolodner associé cette fois à M. Liskay, Université d'Oregon, Portland, USA) ont publié la découverte d'un deuxième gène, nommé hMLHI (human mutL homologue 1) responsable de $30 \%$ des cas héréditaires de cancer du côlon (HNPCC) [11, 12].

À la recherche de ce second gène d'HNPCC, qui présentait à nouveau un phénotype d'instabilité des séquences microsatellites, les chercheurs du groupe de l'université J. Hopkins ont choisi la voie express. Encouragés par la très forte conservation des gènes du SRM, ils ont directement effectué une recherche dans la banque (privée) d'ADNc de la société HGS 
(Human (jenome Sciences), société commerciale dérivée du TIGR (The Institute for (ienomic Research) animé par Craig Venter (Gaithersburg, MI), USA), qui contient près de 100000 séquences. Cette recherche "par téléphone " permit d'identifier trois candidats homologues au mutL bactérien. L'un d'entre eux, $h M L H 1$, se trouvait être localisé sur le chromosome 3, à l'emplacement du deuxième gène HNPCC. Suivant les familles, le gène $h M L H 1$ ou le gène hMSH2 est muté chez les patients HNPCC (à l'état hétérozygote dans les cellules somatiques et homozygotes fonctionnels dans les tumeurs). Les extraits d'une lignée cellulaire issue de ces tumeurs ont montré un défaut de réparation de molécules contenant des mésappariements, confirmant les attentes [13]. Pris ensemble, $h M L H 1$ et hMSH2 expliquent $90 \%$ des cas de HNPCC ; il serait intéressant de savoir si les autres homologues mutL identifiés par l'équipe de l'université J. Hopkins, (hPMS1 et hPMS2), ou d'autres gènes de réparation pourraient expliquer les $10 \%$ restants.

\section{Maladies génétiques et instabilité de l'ADN}

Le HNPCC est l'une des maladies génétiques les plus fréquentes (une personne sur 200). Même si la mutation est récessive au niveau d'une cellule, sa forte pénétrance au niveau somatique la fait apparaître comme dominante pour l'individu, probablement à cause de la probabilité élevée d'une homozygotie somatique à l'origine des tumeurs. Malgré cette fréquence élevée du HNPCC, seul l'état hétérozygote a été observé, l'état homozygote étant probablement létal. Les expériences chez les souris transgéniques permettront de tester cette hypothèse, et fourniront vraisemblablement un bon modèle animal pour le HNPCC. Outre le côlon, les individus HNPCC semblent souffrir d'un risque accru pour d'autres cancers (épithélium et endomètre). Ce n'est pas le seul cas de cancer lié à des instabilités au niveau des microsatellites (citons pour exemple des cas sporadiques
[14], du pancréas et de l'estomac [15] et, à un degré moindre, des cancers du sein ou des ovaires [16]). Une instabilité des séquences microsatellites dans des cas sporadiques de cancer suggère fortement un lien avec un défaut de réparation. Le lien entre la prédisposition à ces différents cancers, la progression de la tumeur et les mutations dans les gènes du SRM devrait permettre une meilleure compréhension au niveau moléculaire de la cancérogenèse. Aucun argument irréfutable ne permet d'expliquer l'apparente spécificité (côlon proximal) des cancers liés à $h M S H 2$ ou $h M L H 1$. Néanmoins, on peut proposer des hypothèses. Par exemple, des agents qui auraient cette spécificité métabolique tissulaire et produiraient des lésions de l'ADN reconnues par MutS (notamment les agents alkylants ou intercalants) pourraient être responsables de ces cancers spécifiques. On peut aussi supposer qu'une cellule défectueuse en SRM ait besoin d'un environnement intracellulaire particulier (par exemple, une mutation dans un oncogène particulier l'immortalisant, ou un contexte tissulaire favorisant) pour devenir cancéreuse [17].

En plus des cancers mentionnés cidessus, d'autres maladies génétiques sont liées à des instabilités génétiques des séquences de type microsatellite. Ainsi, des expansions importantes de triplets (CXG) été observées dans plusieurs maladies neurologiques et neuromusculaires (sept à cette date, dont la chorée de Huntington et l'X fragile) [18] $\left(\mathrm{m} / \mathrm{s} n^{\circ} 4\right.$, vol. 10, p. 472). Bien que l'on ignore les mécanismes qui expliquent ces grandes expansions, un défaut dans la correction des brins lors de la réplication est vraisemblable. Il est donc possible que, grâce aux avancées de la recherche sur les gènes de réparation de l'ADN, d'autres loci associés à des maladies génétiques soient découverts.

Par exemple, certaines maladies mitochondriales, caractérisées par des délétions, des duplications ou l'accumulation de mutations ponctuelles [19] pourraient être liées à des mutations dans le SRM. Comme décrit ci-dessous, les levures mutées dans le gène $\mathrm{MSHI}$ ( mutS homologue l, codant pour une protéine mitochondriale) présentent un phénotype "petite", caractérisé par des mutations de l'ADN mitochondrial (ADNmt) [20]. Étant donné le nombre d'homologues spécialisés du SRM chez les eucaryotes, le dysfonctionnement des différents systèmes humains de réparation des mésappariements pourrait être responsable de nombreuses maladies génétiques (figure 1).

Les phénotypes du système de réparation des mésappariements (SRM) témoignent de fonctions génétiques multiples

La réparation des mésappariements a été postulée, il y a trente ans, afin d'expliquer certaines particularités dans la recombinaison génétique, telles que les ségrégations non mendéliennes des marqueurs génétiques (conversions géniques, etc.), ou l'excès de recombinaison entre mutations rapprochées [21, 22]. La preuve moléculaire confirmant que tout effet de la nature moléculaire de la mutation sur la fréquence de sa recombinaison avec une autre mutation (effet " marqueur") est le résultat de deux mécanismes différents de la réparation des mésappariements a été obtenue en 1987 avec un système utilisant le phage lambda et son hôte E. coli [23]. A cause de l'effet mutateur des mutants hex (mutants ne montrant pas l'effet marqueur dans la recombinaison) de Streptococcus pneumoniae [24-26], la question suivante se posait : comment une réparation des erreurs de réplication peut-elle être dirigée sur le brin néosynthétisé ? Meselson proposa en 1975 que l'état transitoirement hémi-méthylé de l'ADN nouvellement répliqué signalerait l'identité des brins (voir [27] pour une revue historique); un an plus tard, à l'aide d'un ADN hétéroduplex hémi-méthylé, la première preuve expérimentale fut obtenue $[28,29]$. L'isolement et la caractérisation des mutants mutS, mutL et mutH [30] allaient confirmer cette 
première observation. Plusieurs laboratoires déterminèrent la spécificité du système généralisé de réparation des mésappariements bactérien [5, $31]$, un test de réparation in vitro fut publié en 1983 [32], et le groupe de Paul Modrich à l'université de Duke aux États-Unis réussit à purifier les protéines impliquées et à reconstituer in vitro le système de réparation en 1989 [33].

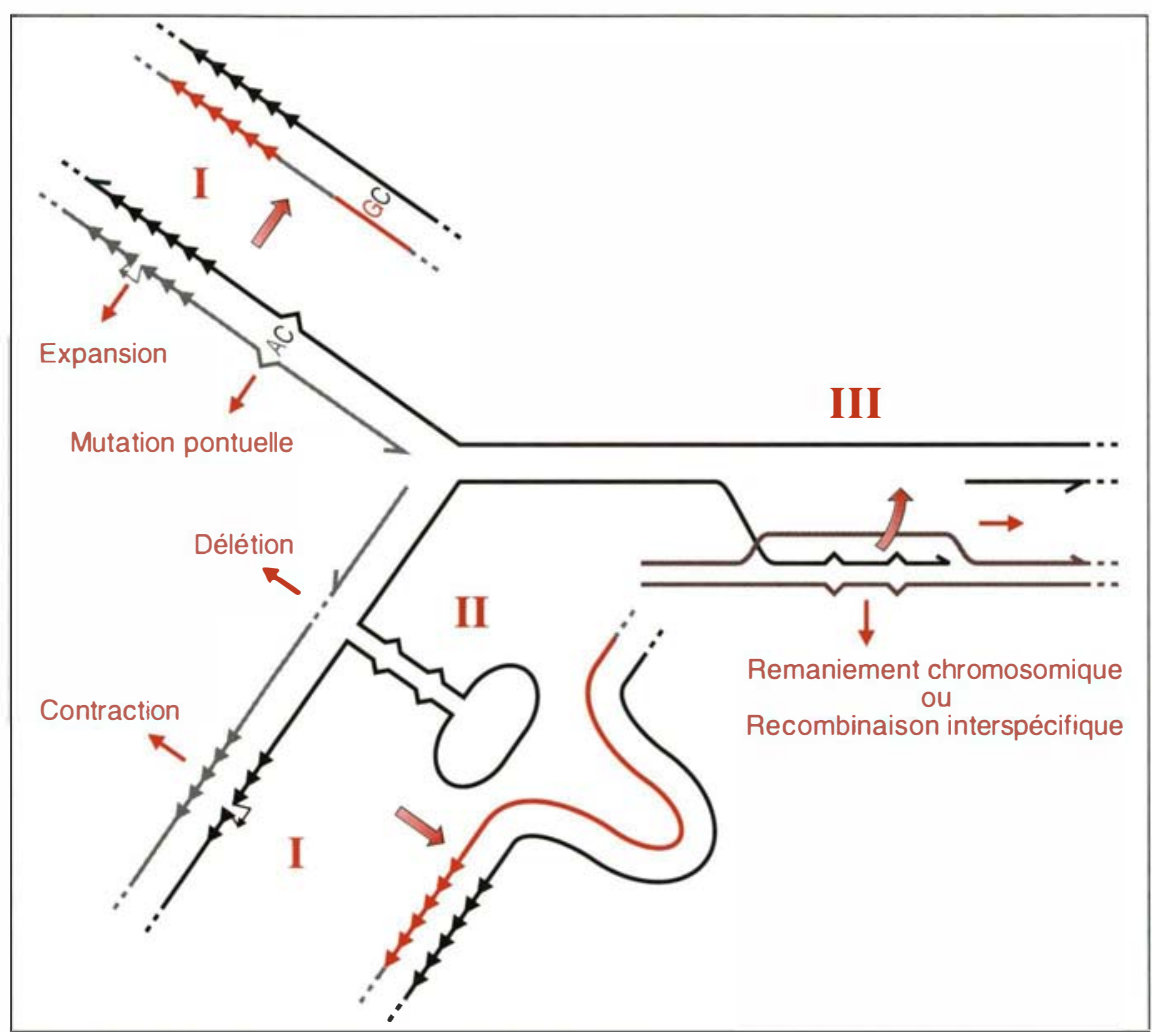

Figure 2. Contrôles de l'intégrité de l'information génétique par le système de réparation des mésappariements (SRM) dE. coli. En noir : brins parentaux; en rouge: synthèse réparatrice ; en gris: brin néosynthétisé; en bistre : ADN partiellement identique. Les flèches épaisses indiquent le résultat de l'action du SRM. Petites flèches : petites répétitions de mono-, di-, trinucléotides. Le "champignon" : structure secondaire (formée par exemple par un transposon). I. Correction (editing) du brin néosynthétisé : prévention des mutations ponctuelles et des petites insertions et délétions par excision de l'ADN néosynthétisé contenant des mésappariements ou des non- appariements. II. Editing du brin matrice: prévention de la formation de délétion par "déroulement " des structures secondaires "délétogènes" contenant des mésappariements. III. Editing de la recombinaison: prévention de la recombinaison entre séquences divergentes, évitant les réarrangements chromosomiques et la recombinaison inter-spécifique. Les conséquences pour la spéciation et la prévention des échanges horizontaux de gènes sont discutées dans [38]. La protéine MutS détecte les mésappariements et non-appariements des bases. La protéine MutL se lie au complexe auquel viennent se joindre I'hélicase II (codée par mutU) et la protéine MutH qui clive le brin néosynthétisé au site GATC non méthylé. Le brin parental est méthylé . cientes en MutS, MutL, ou MutU montrent une augmentation des événements génétiques, tels que : (a) perte d'éléments transposables (phénotype Tex) [34] ; (b) changements de phase de lecture et substitutions de base (phénotype mutateur) (pour revue voir [2, 4, 35] ; (c) grands réarrangements chromosomiques dus à des crossing-over entre des séquences répétées peu divergentes (phénotypes d'instabilités chromosomiques) $[37,38]$; (d) recombinaison de marqueurs génétiques (phénotype hyper-rec) [39], et (e) recombinaison avec une espèce voisine (Salmonella typhimurium) et $E$. coli dont les séquences nucléiques divergent de $15 \%$; phénotype de barrière entre les espèces, lien possible avec la spéciation [40]). La fréquence de ces événements chez les bactéries déficientes par rapport aux bactéries sauvages augmente de 10 et 10000 fois suivant les phénotypes. Tous ces phénotypes sont dus à une incapacité du SRM à opérer sur son substrat, l'ADN mésapparié (voir figure 2). Les délétions résultent de l'enjambement de structures secondaires (en épingle à cheveux), sur le brin matriciel. Lorsque le SRM est fonctionnel, ces structures, si elles contiennent des mésappariements, sont vraisemblablement défaites par l'hélicase II. L'instabilité chromosomique, le caractère hyper-rec et la barrière génétique sont dus à une incapacité de reconnaître les intermédiaires de recombinaison contenant des bases mal appariées dans les régions d'ADN hybride (figure 2). Le phénotype mutateur est empêché dans les bactéries sauvages de façon à préserver la séquence originale. La réparation est dirigée sur le brin néosynthétisé reconnu, soit parce qu'il est non méthylé (la protéine MutH reconnaissant les séquences GATC non méthylées), soit parce qu'il présente des discontinuités (pour revue voir $[2,4]$ ). Comme nous l'avons mentionné précédemment, le SRM bactérien est très conservé chez les eucaryotes comme la levure, l'homme, voire même les plantes (Cerovic et Radman, résultats non publiés). La différence principale entre ces espèces est que les gènes du SRM des eucaryotes sem- 
blent être spécialisés : certains interagissent avec l'ADN mitochondrial, d'autres avec l'ADN nucléaire. Ainsi, les colonies de levures mshl présentent un phénotype "petite ", phénotype qui persiste même après la restauration d'un gène $M S H 1$ fonctionnel. De plus les mitochondries "petites" ainsi engendrées ont un phénotype invasif, c'est-à-dire quasi dominant [20]. Les mutants msh2 et pms 1 montrent, en mitose, un phénotype mutateur et, en méiose, un phénotype hyper-rec [6, 20, 41, 42]. Les mutants $m s h 3$ présentent un faible phénotype mutateur mais semblent augmenter la recombinaison entre séquences divergentes [43]. Le gène $\mathrm{MSH} 4$ est exprimé uniquement en méiose, le phénotype des mutants est à l'étude (S. Roeder, communication personnelle).

\section{Organismes modèles et maladies génétiques}

Les nombreuses données, issues des recherches sur les organismes modèles que sont la bactérie Escherichia coli et la levure Saccharomyces cerevisiae, et la conservation des systèmes de réparation des mésappariements, permettent de faire un certain nombre d'hypothèses sur les implications à attendre chez l'homme. Outre le phénotype mutateur prédit, puis démontré dans le cas de la prédisposition aux cancers du côlon, on peut s'attendre à voir le système de réparation des mésappariements jouer un rôle dans de nombreuses maladies. Ainsi, l'augmentation des réarrangements chromosomiques observée dans des maladies telles que l'anémie de Fanconi ou le cancer du sein, pourrait être liée à un défaut du SRM dans la correction (editing) de la recombinaison. Les délétions entre éléments répétés inversés (tels que les Alu ou les LINE) pourraient être liées au phénotype "Tex" décrit ci-dessus. Le vieillissement, en particulier dans des cas comme la progérie, pourrait être lié au SRM de l'ADN mitochondrial, comme le laisse penser le phénotype "petite" des levures mutées gène de levure (MSH4), exprimé spécifiquement lors de la méiose, suggère un rôle dans la fertilité, voire au niveau de la barrière génétique entre espèces proches dont les croisements sont stériles.

La spécificité moléculaire de reconnaissance des mésappariements et non-appariements de bases par la protéine MutS permet, d'une part, d'utiliser cette protéine afin de diagnostiquer les mutations ponctuelles (après formation d'un hétéroduplex entre un ADN "sauvage " et un $\mathrm{ADN}$ à tester car MutS ne se lie que si l'ADN à tester est muté [44]), et, d'autre part, d'expliquer les instabilités des séquences microsatellites. Ainsi, les microsatellites, si utiles pour la cartographie du génome humain, ont une stabilité variable. Dans les cellules "normales", les répétitions de tétranucléotides sont apparemment les plus instables [45] ; or, les glissements de la polymérase sont plus fréquents sur les répétitions de mono, di ou trinucléotides. La protéine MutS reconnaît très bien les petites boucles, jusqu'à trois nucléotides, et assez mal celles de quatre nucléotides. Le degré de l'effet "accordéon ", observé sur les microsatellites dans les cellules sauvages, est donc dû aux glissements des polymérases et à la correction dépendante de MutS. Dans les cellules cancéreuses HNPCC, où le SRM n'est pas fonctionnel, les répétitions de mono, di ou trinucléotides sont déstabilisées mais pas les tétranucléotides (qui sont peu reconnus par mutS).

De manière générale, le système de réparation des mésappariements est un des éléments essentiels du contrôle négatif des altérations génétiques (figure 3). Celles-ci sont responsables de maladies héréditaires lorsqu'elles ont lieu dans la lignée germinale, et de cancers lorsqu'elles ont lieu dans la lignée somatique. Afin d'éviter ces altérations délétères, les êtres vivants disposent de mécanismes nombreux et sophistiqués de prévention et de réparation des altérations génétiques [46].

Néanmoins, sans mutation, point de diversité génétique et point d'adaptation à un environnement changeant ; les altérations génétiques res-

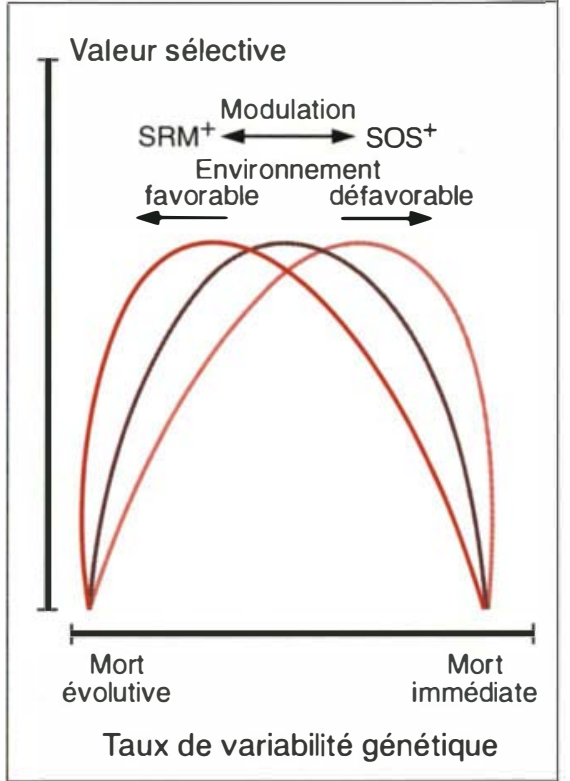

Figure 3. Altérations génétiques et évolution. De nombreux gènes étant indispensables à la survie, trop de mutations induisent des maladies génétiques ou des cancers, voire même une incapacité à se reproduire (" mort immédiate "). Trop peu de mutations empêchent l'adaptation à un nouvel environnement entraînant une mort évolutive. Les altérations génétiques apparaissent donc comme le prix à payer pour l'évolution. La diversité génétique est finement modulée par un couple antagoniste, les systèmes du SOS et de réparation des mésappariements. Ce dernier est constitutif et empêche l'apparition de mutation. Le système SOS, créateur de variabilité, est inductible permettant l'adaptation aux conditions défavorables. II existe donc un taux de variabilité génétique qui optimise la valeur sélective (fitness). Cette valeur optimale dépend de l'adaptation de l'individu à son environnement et varie donc avec les changements d'environnement, d'où la nécessité d'une modulation du taux de variabilité génétique dans la population. 
ponsables de maladies sont donc le prix à payer pour notre capacité d'évoluer. Pour minimiser ce prix, l'évolution a sélectionné des systèmes inductibles de création de variabilité génétique, véritable contrôle positif des altérations génétiques (figure 3). L'induction de ces systèmes mutateurs, dont le plus étudié est le système SOS bactérien [47, 48], augmente "à la demande" l'ensemble des altérations génétiques que le système de réparation des mésappariements réprime (figure 2). Un système inductible présente de nombreux avantages : dans un environnement favorable, le taux de mutation est très faible ; en revanche, en situation de stress (due à des rayonnements, des agents chimiques, une carence, etc.), le système SOS est induit, augmentant le taux de variabilité génétique et donc l'adaptabilité. Ainsi, le système SOS, indispensable à l'évolution, pourrait être, en réponse à des facteurs environnementaux, à l'origine des très nombreux cas sporadiques de maladies génétiques. Le syndrome de Bloom, dans lequel sont produites de manière constitutive une série de protéines inductibles, pourrait être dû à un système de type SOS qui, d'inductible, serait devenu constitutif, ce qui expliquerait le fort taux d'instabilité génétique et de cancers observés chez ces patients $\left(\mathrm{m} / \mathrm{s} n^{\circ} 5\right.$, vol. 3, p. 304, [49-51]). De façon analogue à la recherche sur le système de réparation des mésappariements, la compréhension des mécanismes d'induction des systèmes apparentés au SOS bactérien devrait permettre de diriger la recherche, af in de comprendre les liens existants entre environnement et maladie génétique. Grâce à la conservation considérable des mécanismes de base du métabolisme de l'ADN de la bactérie à l'homme, la génétique bactérienne restera une source de nouveaux paradigmes indiquant des directions pour la recherche des causes des altérations génétiques chez l'homme. La découverte qu'un défaut du système de réparation des mésappariements humains prédispose au cancer du côlon est l'exemple le plus récent d'une telle recherche ciblée

\section{RÉFÉRENCES}

1. Service RF. Stalking the start of colon cancer. Science 1994 ; 263 : 155960.

2. Claverys JP. Correction des mésappariements et stabilité de l'information génétique. médecine/sciences $1992 ; 8$ : 1-11.

3. Peltomaki P, Aaltonen LA, Sistonen P, Pylkkanen L, Mecklin JP, Jarvinen H, Green JS, Jass JR, Weber JL, Leach FS, Peterson GM Hamilton SR, de la Chapelle A, Vogelstein B. Genetic mapping of a locus predisposing to human colorectal cancer. Srience $1993 ; 260$ : $810-2$.

4. Modrich P. Mechanism and biological effects of mismatch repair. Annu Rev Genet $1991 ; 25: 229-53$.

5. Radman M, Wagner $\mathbf{R}$. Mismatch repair in Escherichia coli. Annu Rev Genet 1986 ; 20 : 523-38.

6. Reenan RAG, Kolodner RD. Isolation and characterization of two Saccharomyces cerevisiae genes encoding homologs of the bacterial HexA and MutS mismatch repair proteins. Genetics $1992 ; 132$ : 963-73.

7. Strand M, Prolla TA, I iskay RM, Petes TD Destabilization of tracts of simple repetitive DNA in yeast by mutations affecting DNA mismatch repair. Nature $1993 ; 365: 2746$.

8. Kahn A. Génétique du cancer colo-rectal. médecine/sciences $1994 ; 10: 228-9$.

9. Fishel R, Lescoe MK, Rao MRS, Copeland NG, Jenkins NA, Garber J, Kane M, Kolodner $R$. The human mutator gene homolog $\mathrm{MSH} 2$ and its association with hereditary nonpolyposis colon cancer. Cell $1993 ; 75: 1027-38$.

10. Leach FS, Nicolaides NC, Papadopoulos N, Liu B, Jen J, Parson R, Peltomaki P. Sistonen P, Aaltonen IA, Nystrom I ahti M, Guan XY, Zhang J, Meltzer PS, WU JW, Kao FT, Chen DI, Cerosaletti KM, Fournie REK, Todd S, Lewis T, Leach RJ, Naylor SL, Weissenbach $J$, Mecklin JP, Jarvinen $H$ Peterson GM, Hamilton SR, Green J, Jass J Watson P, Lynch HT, Trent JM, de la Chapelle A, Kinzier KW, Vogelstein B. Mutations of a muts homologue in hereditary nonpolyposis colorectal cancer. Cell 1993; $75: 1215-26$.

11. Bronner CE, Baker SM, Morrison PT Warren G, Smith LM, Lescoe MK, Kane M, Earabino C, Lipford J, Lindlom A, Tannergaid P, Bollag RJ, Godwin AR, Ward DC, Nordenskjold M, Fishel R, Kolodner $\mathbf{R}$ Liskay RM. Mutation in the DNA mismatch repair gene homolog $h M L H I$ is associated with hereditary non-polyposis cancer. with hereditary non-pol
Nature $1994 ; 368: 258-61$.

12. Papadopoulos N, Nicolaides NC, Wei YF, Ruben SM, Carter KC, Rose CA Haseltine WA, Fleishmann RD, Fraser CM Adams MD, Venter JC, Hamilton SR, Petersen GM, Watson P, Lynch HT Peltomaki P, Mecklin JP, de la Chapelle A, Kinzier KW, Vogelstein B. Mutation of a mutL homolog in hereditary colon cancer Science 1994 ; 263 : 1625-9.

13. Parsons R, Li GM, Longley MJ, Fang W, Papadopoulos $N$, Jen J, de la Chapelle $A$ Kinzier KW, Vogelstein B, Modrich P. Hypermutability and mismatch repair deficiency in RER+ tumor cells. Cell 1993 ; $75: 1227-36$.

14. Gonzalez-Zulueta M. Microsatellite instability in bladder cancer. Cancer Res $1993 ; 53: 5620-3$.

15. Han HJ, Yanagisawa A, Kato Y, Park JG, Nakamura $Y$. Genetic instability in pancreatic cancer and poorly differentiated type of gastric cancer. Cancer Res $1993 ; 53$ : 5087-9.

16. Wooster R, Cleton-Janson AM, Collins $\mathrm{N}$, Mangion J, Cornelis RS, Cooper CS, Gusterson BA, Ponder BAJ, Deimling AV, Wiestler OD, Cornelisse CJ, Devilee $\mathbf{P}$ Stratton MR. Instability of short tandem repeats (microsatellites) in human cancers. Nature Genet 1994 ; 6 : 152-6.

17. Radman $M$, Wagner R. Missing mismatch repair. Nature 1993 ; $366: 722$.

18. Nelson DI, Warren T. Trinucleotide repeat instability: when and where? Nature Genet $1993 ; 4: 107-8$.

19. Schapira AHV. Mitochondrial disorders. Curr Op Genet Dev 1993; 3 : 457-65.

20. Reenan RAG, Kolodner RD. Characterization of insertion mutations in the Saccharymoces cervisiae $\mathrm{MSH} 1$ and $\mathrm{MSH} 2$ genes : evidence for separate mitochondrial and nuclear functions. Genetics $1992 ; 132$ : 975-85.

21. Holliday R. A mechanism for gene conversion in fungi. Genet Res $1964 ; 5: 282$. 304.

22. Ephrussi-Taylor H, Sicard AM, Kamen $\mathrm{R}$. Genetic recombination in DNA-induced transformation of pneumococcus. Genetics $1965 ; 51: 455-75$.

23. Jones $\mathrm{M}$, Wagner $\mathrm{R}$, Radman $\mathrm{M}$. Mismatch repair and recombination in $F$ coli. Cell 1987; $50: 621-6$.

24. Lacks SA. Integration efficiency and genetic recombination in pneumococcal transformation. Genetics $1966 ; 53: 207-35$.

25. Iacks SA. Mutants of Diplococclus pneumoniae that lack deoxyribonucleases and other activities possibly pertinent to genetic transformation. I Bacteriol 1970 ; $101: 373-83$.

26. Tiraby JG, Fox MS. Marker discrimination in transformation and mutation of pneumococcus. Proc Natl Acad Sci USA $1973 ; 70: 3541-5$.

27. Radman M, Wagner R. Mismatch recognition in chromosomal interactions and speciation. Chromosoma 1993 ; 102 : 369 73.

28. Radman M, Villani B, Boiteux $S$, Kinsella AR, Glickman BW, Spadari S. Replication fidelity : mechanisms of mutation avoidance and mutation fixation. Cold Spring Harbor Symp Quant Biol 1979 ; $43: 937-46$

29. Radman M, Wagner RE, Glickman BW, Meselson M. DNA methylation, mismatch connection and genetic stability. In : Alacevic M, ed. Progress in environmental mutagenesis. Amsterdam : Elsevier North Holland, 1980: 261-73. 
30. Glickman BW, Radman M. E. coli mutator mutants deficient in methylationinstructed DNA mismatch correction. Proc Natl Acad Sci USA 1980; 77 : 1063-7.

31. Claverys JP, Lacks SA. Heteroduplex deoxyribonucleic acid base mismatch repair in bacteria. Microbiol Rev 1986 ; 133-65.

32. Lu AL, Clark S, Modrich P. Methyldirected repair of DNA basepair mismatches in vitro. Proc Natl Acad Sci USA $1983: 80: 4639-43$.

33. Lahue RS, Au KG, Modrich P. DNA mismatch correction in a defined system. Science $1989 ; 245: 160-4$.

34. Lundblad V, Kleckner N. Mismatch repair mutations of Escherichia coli $\mathrm{K} 12$ enhance transposon excision. Genetics 1985 ; 109:3-19.

35. Levison G, Gutman GA. High frequencies of short frameshifts in polyCA/TG tandem repeats borne by bacteriophage M13 in Escherichia coli K-12. Nucleic Acids Res 1987; 15 : 5323-38.

36. Petit M, Dimpfl AJ, Radman M, Echols H. Control of large chromosomal duplications in Escherichia coli by the mismatch repair system. Genetics $1991 ; 129$ : 327-32.

37. Radman M, Wagner R, Kricker MC. Homologous DNA interactions in the evolution of gene and chromosome structure. Genome Analysis $1993 ; 7$ : 139-52.

38. Feinstein SI, Low KB. Hyperrecombining recipient strains in bacterial conjugation. Genetics $1986 ; 113: 13-33$.

39. Rayssiguier C, Thaler DS, Radman M. The barrier to recombination between Escherichia coli and Salmonella typhimurium is disrupted in mismatch repair mutants. Nature 1989 ; 342 : 396-401.

40. Williamson M S, Game JC, Fogel S. Meiotic gene conversion mutants in Saccharomyces cerevisiae. I : Isolation and characterization of pmsl-1 and psml-2. Genetics $1985 ; 110$ : 609-46.

41. Kramer W, Kramer B , Williamson MS , Fogel S. Cloning and nucleotide sequence of DNA mismatch repair gene PMS from Saccharomyces cervisiae : homology of PMS1 to procaryotic MutL and HexB. J Bacteriol $1989 ; 171: 5339-46$

42. New L, Liu K, Crouse GF. The yeast gene $M S H 3$ defines a new class of eukaryotic MutS homologues. Mol Gen Genet 1993 ; 239 : 97-108.

43. Wagner R, Radman M. A method for detection of mutations using mismatch binding proteins. Eur Patent PCT Pub 1991 Feb 1993 .

44. Weber JL, Wong C. Mutation of human short tandem repeats. Hum Mol Genet 1993 ; 2:1123-8.

45. Taddei F, Radman M. Evolution de l'ADN. In : Tort P, ed. Dictionnaire de l'Evolution et du Darwinisme. Paris : Presses Universitaires de France, 1994 (sous presse)

46. Radman M. SOS repair hypothesis: phenomenology of an inducible DNA mutagenesis. In : Hanawalt P, Setlow RB, eds. Molecular mechanisms for repair of DNA part A. New York : Plenum Publishing Corp, $1975: 355-67$.

47. Devoret R. Mécanisme de la mutagenèse SOS chez les bactéries. médecine/sciences 1993 ; $9: 1-7$.

48. Herrlich P. 12-0-Tetradecanoylphorbol 13-acetate-inducible proteins are synthesized at an increased rate in Bloom syndrome fibroblasts. Proc Natl Acad Sci USA 1982; 79 : 7886-90.

49. Herrlich P, Rahmsdorf HJ, Mallick U, Ponta H, Heiber L, Lucke-Hule C, Koch N. Radiation and mutagen inducible mammalian genes. Biochimie $1982 ; 64: 707-8$. 50. Herrlich P, Mallick U, Ponta $\mathrm{H}$ Rahmsdorf HJ. Genetic changes in mammalian cells reminiscent of an SOS response. Hum Genet 1984; $67: 360-8$.

51. Benditt EP, Benditt JM. Evidence for a monoclonal origin of human atherosclerotic plaques. Proc Natl Acad Sci USA $1973 ; 70: 1753-6$.

\section{Miroslav Radman}

Directeur de recherche au Cnrs.

François Taddei

Ingénieur du génie rural, des eaux et des forêts.

Jennifer Halliday

Chercheur au Cnrs.

Laboratoire de mutagenèse, Institut

Jacques-Monod, 2, place Jussieu, 75251 Paris Cedex 05, France.

\section{TIRÉS A PART}

